\title{
Anemia Infecciosa Equina na Região Norte do Brasil no Período 2005-2017
}

\author{
Taiã Mairon Peixoto Ribeiro $^{1 *} \&$ Lucas Marlon Freiria $^{2}$
}

1. Universidade Federal do Tocantins. (UFT). Campus Araguaína, Escola de Medicina Veterinária e Zootecnia, BR -153, km 112, Zona Rural, CEP 77800-000, Araguaína, TO, Brasil.

* E-mail para correspondência: ribeiro.vet@uft.edu.br

2. Instituto Federal de Rondônia. (IFRO). Campus Cacoal. BR-364, km 228, Lote 2, Zona Rural, CEP 76.960-970, Cacoal, RO, Brasil.

Recebido em: 14 de abril e de 2018. Aceito em: 10 de julho de 2018. Publicado PDF em: 18 de julho de 2018

\section{RESUMO}

Anemia Infecciosa Equina na Região Norte do Brasil no Período 2005-2017. A anemia infecciosa equina constitui uma das doenças mais relevantes em membros desta espécie devido ao prejuízo aos produtores. A Região Norte possui características climáticas e ambientais propícias para a criação de herbívoros, contudo estas mesmas características também favorecem aos insetos vetores desta enfermidade. O presente trabalho teve como objetivo analisar a quantidade de casos notificados de Anemia Infecciosa Equina na Região Norte do Brasil no período de 2005 a 2017. Os dados foram obtidos do Sistema Nacional de Informação Zoossanitária do Ministério da Agricultura, Pecuária e Abastecimento. Foram constatados 19.239 casos no período estudado, com uma média de 1.480 casos anuais. Não houve uma variação significativa da quantidade anual de casos no período, mas foi observado que os estados com menores rebanhos da região possuíram as maiores proporções em relação ao número de casos/efetivo no ano de 2016. Recomenda-se a adoção de medidas preventivas em relação ao manejo sanitário e controle de vetores e o exame periódico do rebanho como forma de minimizar e controlar o impacto econômico causado por esta enfermidade.

PALAVRAS CHAVE: Equidae; Lentivirus Equino; Retroviridae .

\begin{abstract}
Equine Infectious Anemia in Northern Region of Brazil in the Period 2005-2017. Equine infectious anemia is one of the most relevant diseases in members of the Equidae family due to the injury to producers. The North Region has climatic and environmental characteristics propitious for the creation of herbivores as a reasonable price of land and adequate water availability, however these same characteristics also favor the insects vectors of this disease. The objective of this study was to analyze the number of reported cases of Equine Infectious Anemia in the Northern Region of Brazil between the years of 2005 and 2017. Data were obtained from the National Zoossanitary Information System of the Ministry of Agriculture, Livestock and Food Supply. There were 19,239 cases in the period studied, with an average of 1,480 cases per year. There was no significant variation in the annual number of cases I $n$ the period, but was observed that the states with the lowest herds in the region had the greatest proportions in relation to the number of cases / effective in the year 2016. We recommend the adoption of preventive measures in relation to sanitary management and vector control and the periodic examination of the herd as a way to minimize and control the economic impact caused by this disease.
\end{abstract}

KEY WORDS: Equidae; Equine Lentivirus; Retroviridae. 


\section{INTRODUÇÃO}

A anemia infecciosa equina (AIE) é uma enfermidade causada por um vírus da família Retroviridae e gênero Lentivirus que causa uma infecção persistente em membros da família Equidae (equinos, asininos e muares) em todo mundo, com exceção de determinados locais em que ocorrem técnicas de higiene e que não permitem o desenvolvimento de vetores (Ricotti et al. 2016; Gaudaire et al. 2018; Wang et al. 2018).

A notificação da infecção pelo vírus da anemia infecciosa equina (VAIE) é compulsória e determinada pela Organização Mundial de Saúde Animal (OIE) e pelo Ministério da Agricultura, Pecuária e Abastecimento (MAPA) (Brasil 2013; Cook et al. 2013). No Brasil, a legislação em defesa sanitária animal determina que o equídeo com reação soropositiva na técnica de Imunodifusão em Ágar Gel (IDGA) seja eutanasiado, tendo direito de uma contraprova e um reteste, com exceção do estabelecimento de áreas de alto risco, como o Bioma Pantanal (Brasil 2004).

$O$ veículo de transmissão é o sangue de um equídeo infectado que é transmitido mecanicamente por moscas do gênero Tabanus spp. (mosca do cavalo), Stomoxys calcitrans (mosca dos estábulos) e de forma iatrogênica (uso compartilhado de equipamentos equestres e instrumental veterinário não esterilizado) (Issel \& Foil 1984; Nogueira et al. 2017). Outras formas menos significativas de transmissão incluem a transmissão por meio da ingestão de colostro, via uterina e através do coito (Nogueira et al. 2017).

De acordo com More et al. (2008) pode ocorrer o risco da transmissão via aerossol, no entanto ainda são necessários mais estudos. De acordo com Ricotti et al. (2016) é possível que os equídeos infectados com VAIE possam ser sorologicamente indetectáveis por pelo menos 24 meses (dois anos), apesar da detecção molecular de sequências de ácido nucleico do VAIE, e que a transmissão de pequenas quantidades de partículas virais por grandes tabanídeos possa ocasionar o estabelecimento de infecções indetectáveis no diagnóstico sorológico. A doença pode se manifestar basicamente em três fases distintas que seriam a forma aguda, crônica e assintomática ou inaparente (Franco \& Paes 2011; Santos et al. 2011; Leite et al. 2013; Cruz et al. 2015; Ricotti et al. 2016; Roberts 2017).
Todos os estados do Brasil já registraram oficialmente casos notificados de Anemia Infecciosa Equina, e em particular, na Região Norte, apenas nos estados do Acre, Pará, Rondônia e Roraima foram realizados estudos científicos (Santos et al. 2001; Heinemann et al. 2002; Aguiar et al. 2008; Mattos et al. 2010; Brasil 2017). Na Região Norte já foram observadas prevalências de 1,34 - 46,26\% no Pará, 7,5\% no Acre, 11,76 - 60\% em Roraima e de 1,15 - 9,6 \% em Rondônia (Braga 2000; Santos et al. 2001; Pena et al. 2006; Aguiar et al. 2008; Mattos et al. 2010; Freitas et al. 2015; Guiraud et al. 2017). Ambientes quentes e úmidos como os da Região Amazônica são propícios para a reprodução dos vetores tabanídeos, assim como a elevada pluviometria presente na Região (Heidmann et al. 2012; Santos et al. 2016).

$\mathrm{O}$ presente trabalho objetivou analisar os casos registrados de infecção pelo Vírus da Anemia Infecciosa Equina em Equídeos diagnosticados com o uso dos testes sorológicos recomendados pelo MAPA e OIE na Região Norte do Brasil, por meio de consulta ao Sistema Nacional de Informação Zoossanitária do Ministério da Agricultura, Pecuária e Abastecimento entre os anos de 2005 e 2017.

\section{MATERIAL E MÉTODOS}

O estudo é retrospectivo e descritivo da quantidade de casos diagnosticados de Anemia Infecciosa Equina em equinos, asininos e muares da Região Norte do Brasil. Esta região é composta pelos Estados do Acre, Amazonas, Amapá, Pará, Rondônia, Roraima e Tocantins e possui um efetivo de rebanho equídeo de 897.858 cabeças (Brasil 2016).

Definiu-se como caso, o equídeo doente ou infectado com diagnóstico confirmado por meio do teste de Imunodifusão em Ágar Gel. Foram avaliadas informações constantes na base de dados do Sistema Nacional de Informação Zoossanitária no período de 2005 2017 (Brasil 2017). Os dados obtidos foram armazenados em planilhas eletrônicas por meio do Programa Office Excel 2016®.

\section{RESULTADOS E DISCUSSÃO}

No período entre 2005-2017 foram 
Tabela 1. Casos notificados de AIE em equídeos na Região Norte do Brasil no período 20052017.

\begin{tabular}{|c|c|c|c|c|c|c|c|c|}
\hline Ano & PA & RO & TO & AC & RR & AM & AP & Total \\
\hline 2005 & 168 & 537 & 468 & 171 & 109 & 50 & 0 & 1503 \\
\hline 2006 & 370 & 369 & 496 & 170 & 199 & 25 & 0 & 1629 \\
\hline 2007 & 439 & 405 & 441 & 169 & 203 & 36 & 56 & 1749 \\
\hline 2008 & 244 & 363 & 423 & 200 & 160 & 21 & 12 & 1423 \\
\hline 2009 & 642 & 361 & 268 & 274 & 81 & 73 & 17 & 1716 \\
\hline 2010 & 391 & 337 & 292 & 175 & 34 & 64 & 9 & 1302 \\
\hline 2011 & 698 & 232 & 214 & 156 & 75 & 20 & 0 & 1395 \\
\hline 2012 & 613 & 390 & 42 & 153 & 156 & 41 & 18 & 1413 \\
\hline 2013 & 794 & 388 & 86 & 226 & 165 & 47 & 24 & 1730 \\
\hline 2014 & 742 & 264 & 299 & 196 & 203 & 21 & 7 & 1732 \\
\hline 2015 & 436 & 232 & 326 & 69 & 213 & 37 & 38 & 1351 \\
\hline 2016 & 711 & 125 & 446 & 162 & 177 & 99 & 32 & 1752 \\
\hline 2017 & 308 & 51 & 77 & 38 & 43 & 16 & 11 & 544 \\
\hline Total & $\mathbf{6 . 5 5 6}$ & $\mathbf{4 . 0 5 4}$ & $\mathbf{3 . 8 7 8}$ & $\mathbf{2 . 1 5 9}$ & $\mathbf{1 . 8 1 8}$ & $\mathbf{5 5 0}$ & $\mathbf{2 2 4}$ & $\mathbf{1 9 . 2 3 9}$ \\
\hline
\end{tabular}

notificados 19.239 casos confirmados de Anemia Infecciosa Equina na Região Norte (Tabela 1).

A quantidade de casos tem-se mantido estável no período estudado com uma média de 1.480 casos anuais (Figura 1), com as maiores quantidades absolutas e percentuais de notificações realizadas nos Estados que possuem os quatro maiores rebanhos (Pará, Rondônia, Tocantins e Acre) na Região Norte, pois populações maiores tendem a ter maior ocorrência da exposição ao risco de enfermidade (Rothman et al. 2011).

Isto demonstra que a Anemia Infecciosa Equina continua sendo uma doença endêmica no rebanho de equídeos da Região Norte, e que devem ser executadas medidas sanitárias efetivas para o enfrentamento da doença. Entre as medidas recomenda-se o monitoramento sorológico periódico de todo o rebanho de

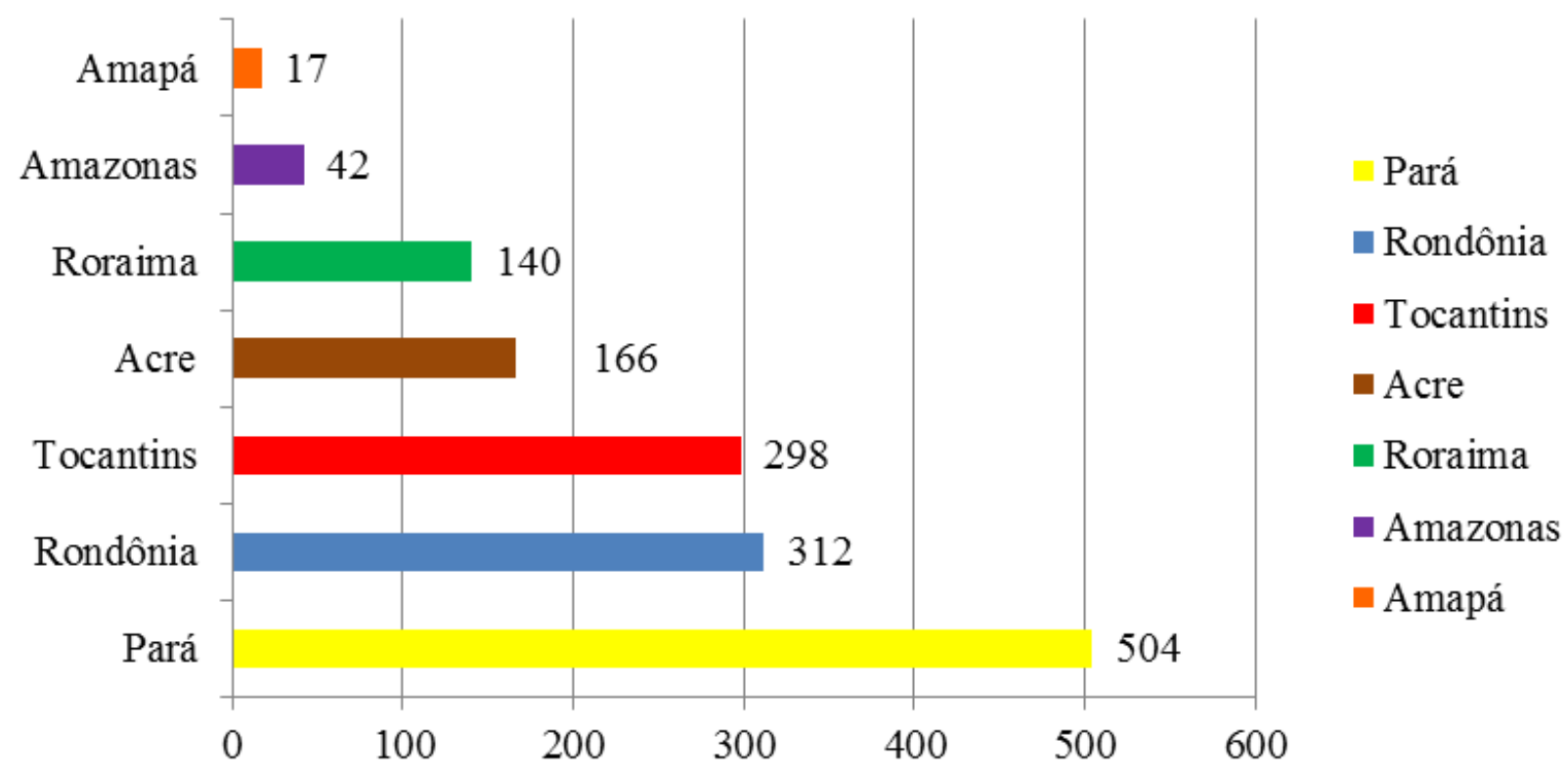

Figura 1. Média anual de casos notificados de AIE em estados da Região Norte no período 20052017. 
equinos, asininos e muares, o não compartilhamento de instrumentos de equitação e a esterilização do material clínico e cirúrgico (Nogueira 2013; Reis 2015). A detecção de animais positivos pelo diagnóstico laboratorial constitui ferramenta essencial ao controle desta enfermidade, pois $95 \%$ dos animais são portadores assintomáticos (Pena et al. 2006).

De acordo com Dias (2000) o alto número de casos de Anemia Infecciosa Equina na Região Norte é devido ao clima e ambiente favorável da Amazônia à ocorrência dos insetos hematófagos transmissores do vírus.

O Estado do Pará, que possui o maior rebanho de equinos da Região Norte, foi responsável por $34 \%$ das notificações realizadas na Região Norte com uma média de 504 casos anuais (Figura 2). Heinemann et al. (2002) ao realizar pesquisa em Uruará-PA, constaram $17,71 \%$ de soropositividade para VAIE. Já nos estudos de Pena et al. (2006), Heidemann et al. (2012) e Freitas et al. (2015) por sua vez constataram, respectivamente, frequências de $1,34 \%, 12,79 \%$ e $46,26 \%$ de sororreatividade para VAIE nas regiões Sul, Centro-sul e na Ilha de Marajó.

Em Rondônia constatou-se 21,12\% dos casos de AIE no período estudado, com uma média de 312 casos anuais. A detecção de equídeos positivos para AIE tem sido relatada em estudos como o de Aguiar et al. (2008) que demonstraram $9,6 \%$ de frequência de soropositividade no município de Monte Negro -RO, e o de Guiraud et al. (2017) que evidenciaram sororreatividade de $1,15 \%$ no ano de 2014 e 1,01\% em 2015 em todas as regiões do estado de Rondônia.

No Estado do Tocantins pôde ser observada frequência $(20 \%)$ semelhante à encontrada no estado de Rondônia com uma média de 298 casos anuais. No Tocantins já foram registradas 34 espécies diferentes de moscas dos cavalos (Diptera: Tabanidae) em região próxima a capital do estado, demonstrando a presença do vetor do VAIE (Lima et al. 2015). A ocorrência de focos de Anemia Infecciosa Equina fez com que a Agência de Defesa Agropecuária do estado do Tocantins (ADAPEC) aprovasse normas complementares ao Programa Estadual de Sanidade de Equídeos (PESE) exigindo a certificação em curso de Resenha, Coleta de Material e Legislação para Exame de AIE e Mormo a partir do ano de 2018 para os Médicos Veterinários cadastrados no PESE (Tocantins 2017).

Roraima foi o estado que teve a maior proporção casos/efetivo para o ano de 2016 $(0,65 \%)$ em relação aos demais estados, e estudos anteriores já demonstraram frequências de soropositivdade variando de $40-60 \%$ no ano de 2.000 e de $11,76 \%$ no ano de 2010 em rebanhos de equídeos no estado (Braga 2000; Mattos et al. 2010). No período estudado, o estado de Roraima teve 9,4\% das notificações

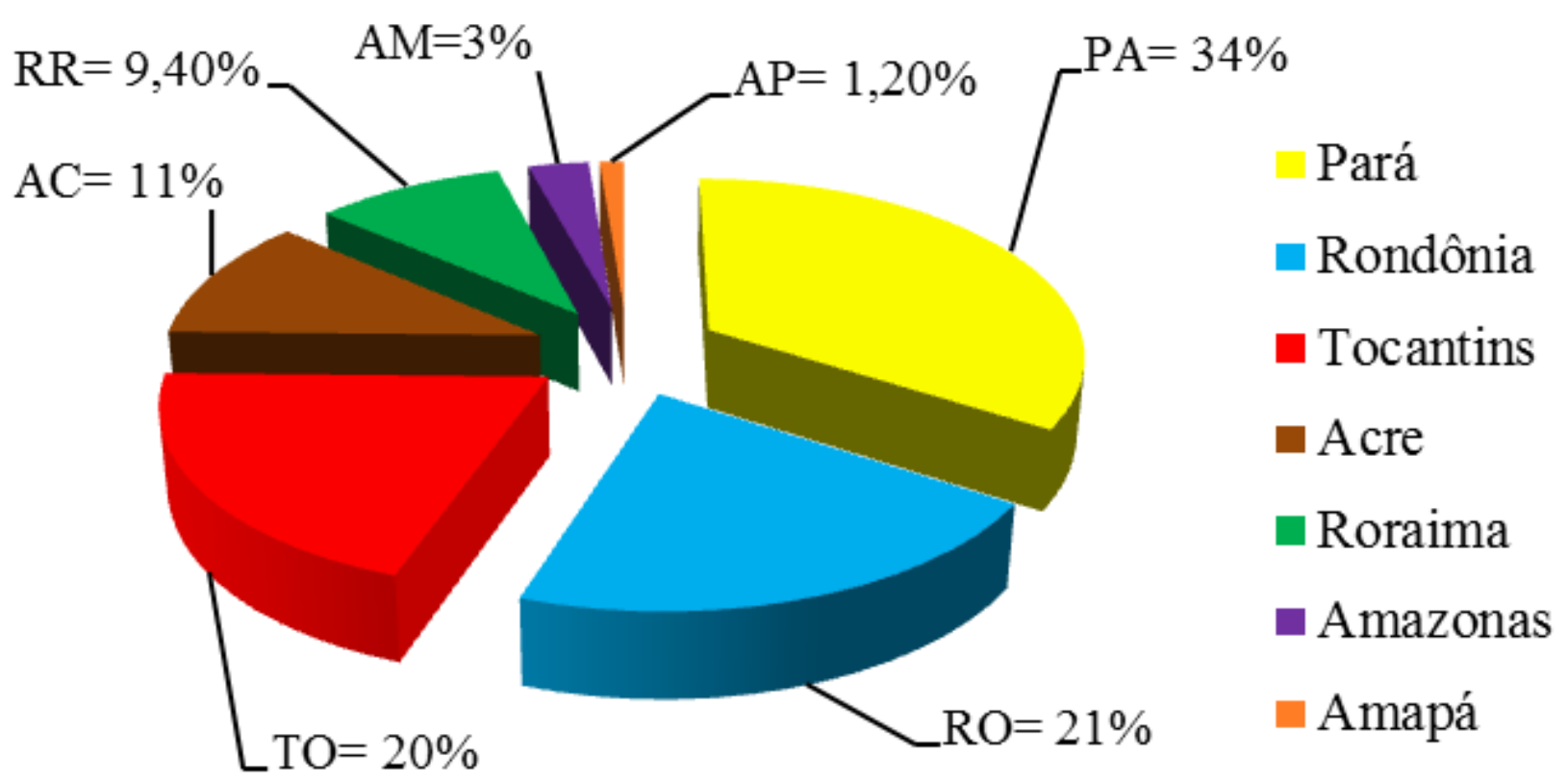

Figura 2. Participação (\%) de cada estado da Região Norte no total de notificações de casos de AIE no período de 2005-2017. 
Tabela 2. Notificações de AIE, efetivo equídeo e proporção Caso/efetivo (\%) de AIE na Região Norte do Brasil no ano de 2016.

\begin{tabular}{|c|c|c|c|c|c|c|c|}
\hline Dados & PA & RO & TO & AC & RR & AM & AP \\
\hline Notificações em 2016 & 711 & 125 & 446 & 162 & 177 & 99 & 32 \\
\hline Efetivo em 2016 & 380.845 & 164.607 & 191.397 & 97.999 & 27.045 & 27.758 & 8.207 \\
\hline Caso/efetivo 2016 (\%) & $0,19 \%$ & $0,08 \%$ & $0,23 \%$ & $0,17 \%$ & $0,65 \%$ & $0,36 \%$ & $0,39 \%$ \\
\hline
\end{tabular}

com uma média de 140 casos anuais.

Do total de notificações foi constatado que $11 \%$ eram pertencentes ao estado do Acre. Observou-se uma média de 166 casos anuais no estado e a sororreatividade para VAIE $(7,5 \%)$ em equídeos já foi constatada por Santos et al. (2001). O Amazonas e Amapá foram responsáveis, respectivamente, por apenas 3\% e $1,2 \%$ das notificações no período. O estado do Amazonas teve uma média de 42 casos anuais e o estado do Amapá teve 17 casos anuais.

A análise das quantidades absolutas pode levar a falsas conclusões se não forem estudadas em conjunto com os dados populacionais do rebanho dos estados. Quando é analisado o coeficiente de casos notificados de 2016 em relação ao efetivo do rebanho de equídeos do mesmo ano, é possível constatar que os estados com as menores quantidades (Roraima, Amazonas e Amapá) de notificações em todo período estudado são os que possuem a maior proporção caso/efetivo (Tabela 2). Nestes estados o número de equídeos positivos é proporcionalmente maior em relação à população total do que os estados com os maiores rebanhos e com as maiores quantidades absolutas de notificações.

Uma maior proporção de notificações em relação ao rebanho total do estado pode sugerir várias hipóteses: favorecimento da transmissibilidade do agente, medidas deficitárias de manejo sanitário animal, alterações das características intraespecíficas do agente etiológico, aumento da quantidade de exames de diagnóstico realizados no período, aplicação de novos testes de diagnóstico ou ainda pela maior mobilização de profissionais de saúde e da população para a notificação de determinada doença.

$O$ custo médio de um equídeo na região amazônica é variável, e soma-se a isto a escassez de estudos nesta área a nível nacional. São estimadas perdas econômicas na faixa de R\$ 57.717.000 (57,717 milhões de reais) durante o período estudado (2005-2017) com a eutanásia dos 19.239 casos de AIE e de R\$ 4.440.000 (4,440 milhões de reais) anuais em relação à média de 1.480 casos anuais, considerando que o preço médio de um equídeo comercializado seja de R\$3.000,00 (três mil reais) em fazendas na Região Norte.

O preço médio do equídeo pode variar em decorrência da caracterização racial, índices produtivos e região estudada, não permitindo, portanto que haja extrapolação de dados de uma região para outra sem que ocorram as devidas ponderações, contudo, podemos observar que o preço médio de um equídeo comercializado em propriedades rurais em Minas Gerais é de R \$ 4.500,00 (quatro mil e quinhentos reais) de diferentes raças e de $\mathrm{R} \$$ 18.000 (dezoito mil reais) para um animal da raça brasileiro de hipismo de 12 meses em São Paulo (Vieira et al. 2015; Santos et al. 2018).

Portanto, o desafio nos estudos de análise de situação de saúde animal são elencar outras variáveis que não são disponíveis pelos meios de comunicação oficiais para que se possa determinar especificamente o quadro da situação da enfermidade em uma população determinada. A maioria dos animais submetidos a teste de diagnóstico pertence a rebanhos em que a doença está controlada devido ao alto valor zootécnico do efetivo, cujos proprietários têm maior esclarecimentos da necessidade do monitoramento sorológico periódico, ou ainda devido a necessidade de movimentação de equídeos em leilões, lazer ou comercialização (Moraes et al. 2017).

São necessários estudos periódicos com amostragem significativa a nível estadual e inclusão de propriedades com diferentes níveis de tecnificação para identificar as áreas com maiores e menores riscos para transmissão de AIE, o que auxiliará na adoção de medidas sanitárias adequadas e na alocação eficiente de recursos humanos e financeiros no combate a tal enfermidade.

\section{CONCLUSÕES}


Constatou-se a endemicidade da Anemia Infecciosa Equina em Equídeos da Região Norte do Brasil, causando prejuízos econômicos devido à eutanásia de animais infectados.

Para o controle da AIE recomenda-se a realização periódica de exames sorológicos para monitoramento do rebanho, o não compartilhamento de equipamentos equestres e a compra de equídeos provenientes de propriedades monitoradas.

\section{REFERÊNCIAS BIBLIOGRÁFICAS}

Assmar, A.C. \& Salles, F.F. 2017. Taxonomic and distributional notes on Spongilla-flies (Neuroptera: Sisyridae) from Southeastern Brazil with first interactive key to the species of the country. Zootaxa 4273: 80-92.

Aguiar, D.M.; Cavalcante, G.T.; Lara, M.C.C.S.H.; Villalobos, E.M.C.; Cunha, E.M.S.; Okuda, L.H.; De Stéfano, E.; Nassar, A.F.C.; Souza, G.O.; Vasconcellos, S.A.; Labruna, M.B.; Camargo, L.M.A \& Gennari, S.M. 2008. Prevalência de anticorpos contra agentes virais e bacterianos em equídeos do município de Monte Negro, Rondônia, Amazônia Ocidental Brasileira. Brazilian Journal of Veterinary Research and Animal Science 45(4): 269276.

Braga, R.M. 2000. Cavalo lavradeiro em Roraima: aspectos históricos, ecológicos e de conservação. Brasília: Embrapa Comunicação para Transferência de Tecnologia, 119p.

Brasil. Ministério de Agricultura, Pecuária e Abastecimento. 2004. Instrução normativa $\mathrm{N}^{\circ} 45$, de 15 de junho de 2004. Aprova as normas para a prevenção e o Controle da Anemia Infecciosa Equina - A.I.E. Diário Oficial da União, Seção 1.

Brasil. Ministério da Agricultura, Pecuária e Abastecimento. Secretaria de Defesa Agropecuária. 2013. Instrução Normativa $N^{\circ}$ 50, de 23 de setembro de 2013. Alterar a lista de doenças passíveis da aplicação de medidas de defesa sanitária animal. Diário Oficial da União. Seção 1.

Brasil. Instituto Brasileiro de Geografia e Estatística. 2016. Produção Pecuária Municipal - Volume. 44. Rio de Janeiro: Instituto Brasileiro de Geografia e Estatística. $44 \mathrm{p}$.

Brasil. Ministério da Agricultura, Pecuária e Abastecimento. 2017. Sistema Nacional de Informação Zoossanitária - Coordenação de Informação e Epidemiologia - Saúde Animal. Ministério da Agricultura, Pecuária e Abastecimento,
Brasília. (http://indicadores.agricultura.gov.br/ saudeanimal/index.htm). Acesso em 14/04/2017.

Cook, R.F.; Leroux, C. \& Issel, C.J. 2013. Equine infectious anemia and equine infectious anemia virus in 2013: a review. Veterinary Microbiology 167(12): 181-204

Cruz, F.; Fores, P.; Ireland, J.; Moreno, M.A. \& Newton, R. 2015. Freedom from equine infectious anemia virus infection in Spanish Purebred horses. Veterinary Record Open 2 (1): e000074.

Dias, H.L.T. 2000. Soroepidemiologia de cinco enfermidades infecciosas em eqüinos criados no Estado do Pará. Tese de Doutorado, Universidade Federal do Pará, Belém. 147 p.

Franco, M.M.J. \& Paes, A.C. 2011. Anemia nfecciosa Equina. Veterinária e Zootecnia 18(2): 197-207.

Freitas, N.F.Q.R.; Oliveira, C.M.C.; Leite, R.C.; Reis, J.K.P.; Oliveira, F.G.; Bomjardim, H.A.; Salvarani, F.M. \& Barbosa, J.D. 2015. Equine infectious anemia on Marajo Island at the mouth of the Amazon river. Pesquisa Veterinária Brasileira 35 (12): 947-950.

Gaudaire, D.; Lecouturier, F.; Ponçon, N.; Morilland, E.; Laugier, C.; Zientara, S. \& Hans, A. 2018. Molecular characterization of equine infectious anaemia virus from a major outbreak in southeastern France. Transboundary and Emerging Diseases 65 (1): e7e13.

Guiraud, A.C.C.; Belo, M.A.A.; Moraes, A.C.; Manrique, W.G.; Souza, L.M.; Charlie-Silva, I. \& Souza, E.P. 2017. Levantamento soroepidemiológico de anemia infecciosa equina no estado de Rondônia, Brasil. Ars Veterinaria 33(1): 31-36.

Heidmann, M.J.; Fischer, V.L; Manhezzo, T.G.; Santos, J.D. \& Castro, B.G. 2012. Estudo retrospectivo da anemia infecciosa equina na região Centro-sul do Pará, Brasil, 2007-2010. Revista Brasileira de Medicina Veterinária 34(3): 192-197.

Heinemann M.B.; Cortez, A.; Souza, M.C.C.; Gotti, T.; Ferreira, F.; Homem, V.S.F.; Ferreira Neto, J.S.; Soares, R.M.; Sakamoto, S.M.; Cunha, E.M.S. \& Richtzenhain, L.J. 2002. Soroprevalência da anemia infecciosa equina, da arterite viral dos equinos e do aborto viral equino no município de Uruará, PA, Brasil. Brazilian Journal of Veterinary Research and Animal Science 39(1): 50-53.

Issel, C.J. \& Foil, L.D. 1984. Studies on equine infectious anemia virus transmission by insects. Journal of American Veterinary Medical Association 184: 293-297.

Leite, R.C.; Reis, J.K.P.; Oliveira, A.P.; Nascimento, P.M.P.; Oliveira, F.G.; Naves, J.H.F.F.; Rodrigues, A.P.S.; Gasparini, M.R.; Alves, F.; Oliveira, C.H.S.; Rajão, D.S. \& Galinari, G.C.F. 2013. Retroviroses dos animais domésticos. Veterinária e Zootecnia 20: 
Lima, H.I.L.; Krolow, T.K. \& Henriques, A.L. 2015. Checklist of horse flies (Diptera: Tabanidae) from Taquaruçu, Tocantins, Brazil, with new records for the state. Check List 11(2): 1596.

Mattos, P.S.R.; Pezente, L.G.; Mattos, K.K.; Braga, R.M. \& Oliveira, N.T. 2010. Ocorrência de Anemia Infecciosa Equina no Estado de Roraima, Amazônia setentrional brasileira. Boa Vista: Embrapa Roraima, $11 \mathrm{p}$.

Moraes, D.D.A.; Gonçalves, V.S.P.; Mota, A.L.A. \& Borges, J.R.J. 2017. Situação epidemiológica da anemia infecciosa equina em equídeos de tração do Distrito Federal. Pesquisa Veterinária Brasileira 37 (10):1074-1078.

More, S.J.; Aznar, I.; Myers, T.; Leadon, D.P. \& Clegg, T.A. 2008. An outbreak of equine infectious anemia in Ireland during 2006: The modes of transmission and spread in the Kildare Cluster. Equine Veterinary Journal 40(7): 709-711.

Nogueira, M.F. 2013. Cuide da sua montaria, previna a anemia: Como prevenir a anemia infecciosa equina, ou AIE. Corumbá: Empresa Brasileira de Pesquisa Agropecuária, 2p.

Nogueira, M.F.; Oliveira, J.M.; Santos, C.J.S.; Petzold, H.V.; Aguiar, D.N.; Juliano, R.S.; Reis, J.K.P.; Abreu, U.G.P. 2017. Equine infectious anemia in equids of Southern Pantanal, Brazil: seroprevalence and evaluation of the adoption of a control programme. Pesquisa Veterinária Brasileira 37(3):227-233.

Pena, L.J.; Pena, D.A.; Barrios, P.R.; Dale, R.; Lamêgo, M.R.A. \& Moraes, M.P. 2006. Levantamento soroepidemiológico da infecção pelo vírus da Anemia Infecciosa Eqüina, da Influenza Eqüina-2 e do Herpesvírus Eqüino-1 em rebanhos do sul do Estado do Pará, Brasil. Brazilian Journal of Veterinary Research and Animal Science 43 (4): 537-542.Reis, J.K.P. 2015. Métodos de diagnóstico e estratégias de controle da anemia infecciosa equina. Ciência Veterinária nos Trópicos 18(2): 58.

Ricotti, S.; Garcia, M.I.; Veaute, C.; Bailat, A.; Lucca, E.; Cook, R.F.; Cook, S.J. \& Soutullo, A. 2016. Serologically silent, occult equine infectious anemia virus (EIAV) infections in horses. Veterinary Microbiology 187: 41-49.

Roberts, H. 2017. Equine infectious anemia in Europe: an ongoing threat to the UK. Veterinary Record 181(17): 442-446.

Rothman, K.; Greenland, S. \& Lash, T. 2011. Epidemiologia Moderna. Porto Alegre: Artmed, $888 \mathrm{p}$.

Santos, R. M. L.; Reis, J. K. P.; Santos, F. G. A. \& Oliveira, I. C. S. 2001. Frequência de anemia infecciosa equina em equinos no Acre, 1986 a 1996. Arquivo Brasileiro de Medicina Veterinária e Zootecnia 53(3): 310-315.
Santos, E.M.; Motta, P.M.C.; Heinemann, M.B.; Leite, R.C. \& Reis, J.K.P. 2011. Avaliação da nested PCR em comparação aos testes sorológicos IDGA e ELISA para o diagnóstico da anemia infecciosa equina. Arquivo Brasileiro de Medicina Veterinária e Zootecnia 63(2): 296-301.

Santos, J.D.; Lourenço, F.J. \& Castro, B.G. 2016. Estudo retrospectivo da anemia infecciosa equina na região médio-norte matogrossense, Brasil, 2006-2011. Revista Brasileira de Medicina Veterinária 38(1): 79 -85 .

Santos, B.E.S.; Brandi, R.A. \& Gameiro, A.H. 2018. Estudo do mercado e produção do cavalo brasileiro de hipismo no estado de São Paulo. Pubvet 12 (2): 111.

Tocantins. Agência de Defesa Agropecuária do Estado do Tocantins. 2017. Instrução Normativa ADAPEC N 4, de 10 de outubro de 2017. Aprova as normas complementares e medidas voltadas ao Programa Estadual de Sanidade dos Equideos no Estado do Tocantins, através do cadastramento de Médicos Veterinários para realização de coleta de material (soro sanguineo) e requisição de exame de Anemia Infecciosa Equina e Mormo. Palmas: Agência de Defesa Agropecuária do Estado do Tocantins. 6 p.

Vieira, E.R.; Rezende, A.S.C.; Lana, A.M.Q.; Barcelos, K.M.C.; Santiago, J.M.; Lage, J.; Fonseca, M.G. \& Bergmann, J.A.G. 2015. Caracterização da equideocultura no estado de Minas Gerais. Arquivo Brasileiro de Medicina Veterinária e Zootecnia 67 (1): 319-323.

Wang, H.; Rao, D.; Fu, X-Q.; Hu, M-M. \& Dong, J-G. 2018. Equine infectious anemia virus in China. Oncotarget 9(1): 1356-1364. 